\title{
LA-UR=-98-106
}

\section{TASK COMPLETION REPORT FOR UPDATE ZERORGH \\ by}

Robert G. Steinke

September 4, 1997

\section{RECEIVED MAY 281998 \\ OSTI}

\section{MASTER}

DISTRABUTION OF THIS DOCUMENT IS UNLIMITE 


\section{DISCLAIMER}

This report was prepared as an account of work sponsored by an agency of the United States Government. Neither the United States Government nor any agency thereof, nor any of their employees, makes any warranty, express or implied, or assumes any legal liability or responsibility for the accuracy, completeness, or usefulness of any information, apparatus, product, or process disclosed, or represents that its use would not infringe privately owned rights. Reference herein to any specific commercial product, process, or service by trade name, trademark, manufacturer, or otherwise does not necessarily constitute or imply its endorsement, recommendation, or favoring by the United States Government or any agency thereof. The views and opinions of authors expressed herein do not necessarily state or reflect those of the United States Government or any agency thereof. 


\section{DISCLAIMER}

Portions of this document may be illegible electronic image products. Images are produced from the best available original document. 


\title{
TASK COMPLETION REPORT FOR UPDATE ZERORGH
}

\author{
Robert G. Steinke
}

\begin{abstract}
Update ZERORGH programs the Namelist-variable multiplier FRGH to the $\rho \cdot g \cdot h$ gravity-head term in all motion equations of TRAC-P Version 5.4.28.
\end{abstract}

\subsection{INTRODUCTION}

The one-dimensional (1D) hydraulic and three-dimensional (3D) VESSEL components in TRAC-P have in their motion (momentum) equations a $\rho \cdot g \cdot h$ gravity-head term where $\rho$ is the fluid phasic density, $g$ is the acceleration-ofgravity constant $\left(9.80665 \mathrm{~m} \mathrm{~s}^{-2}, 32.17405 \mathrm{ft} \mathrm{s}^{-2}\right)$, and $\mathrm{h}$ is the vertical elevation. Knolls Atomic Power Laboratory requested the capability to apply a Namelist-variable multiplier to the $p \cdot g \cdot h$ gravity-head term in all motion equations of TRAC-P. The default value of this multiplier would be unity. Inputting zero would eliminate the effect of the $\rho \cdot g \cdot h$ gravity-head term in the motion equations.

\subsection{UPDATE ZERORGH}

Update ZERORGH and LABPRG input-data file LABRGH, a listing of which is shown in Appendix A, programs the FRGH multiplier to the $\rho \cdot g \cdot h$ gravity-head term in all motion equations of TRAC-P. This is done for the 1D hydraulic components in subroutines FEMOM and TF1DS1 and for the 3D VESSEL component in subroutines FEMOMX, FEMOMY, FEMOMZ, and TF3DS1. Namelist variable FRGH is defined to Namelist data group INOPTS for input in subroutine INPUT, saved in common block CONTRL, initialized to 1.0 in subroutine BLKDAT, and checked that its input value lies in the range of -1000.0 to 1000.0 in subroutine NAMLST.

\subsection{TESTING}

Update ZERORGH, with other pending updates in TRAC-P Version 5.4:28, was tested with six modified cases of the ZIONPWR standard test problem. ZIONPWR is a vertically oriented flow channel modeled by single FILL, PUMP, 1D VESSEL, VALVE, and BREAK components in series with ROD and SLAB HTSTR components providing heat-transfer coupling to the 1D VESSEL component. Results of that testing are shown in Appendix B. Cases 1 and 2 define FRGH $=1.0$ by its internal default value and by Namelist input, respectively. The solution results of both calculations were identical, as expected. Case 3 defines FRGH $=0.0$ by 
Namelist input, and Case 4 defined the PUMP- and VALVE-component GRAV $=0.0$ and VESSEL-component GZC $=0.0$. Both of these cases effectively zero out the $\rho \cdot g \cdot h$ gravity-head term in all motion equations. This orients the flow channel horizontally. Comparing their TRCOUT files showed only differences caused by the output echo of their different input-variable values. The solution results of cases 3 and 4 were identical, as expected. Cases 5 and 6 are similar in form to cases 3 and 4 , respectively, except that in case 5, FRGH $=0.6$; and in case $6, \mathrm{GRAV}=0.6$ and GZC $=$ -0.6 . This corresponds to the flow channel being oriented $36.87^{\circ}$ from horizontal. Again, TRCOUT-file differences were caused by the output echo of their different input-variable values, and the solution results were identical except for one slight difference. Control blocks -12 and -312 had values that differed by 1 in the seventh significant digit of the seventh large edit (of 9 large edits). Different arithmetic operations evaluating the $\rho \cdot g \cdot h$ gravity-head term and computer-storage format round-off are felt to be the cause of this isolated difference in solution output values.

These six cases evaluate the use of Namelist variable FRGH or the GRAV and GZC variables to orient the ZIONPWR flow channel vertical, horizontal, or $36.87^{\circ}$ from horizontal. Both the FRGH value as a multiplier (and FRGH $=1.0$ and its multiplier value incorporated directly into the GRAV and GZC factors of the $\rho \cdot g \cdot h$ gravityhead term of the motion equations) were shown to give the expected identical solutions for three different orientations of the ZIONPWR flow channel.

\subsection{CONCLUSIONS}

Update ZERORGH and its LABPRG input-data file LABRGH program TRAC-P Version 5.4.28 to apply the new Namelist-variable multiplier FRGH to the $\rho \cdot g \cdot h$ gravity-head term in all motion (momentum) equations. Vertical, horizontal, and $36.87^{\circ}$ from horizontal orientations of the ZIONPWR standard test problem were tested. Defining these orientations to FRGH or directly to GRAV or GCZ gave identical solutions in each of the three cases.

\section{ACKNOWLEDGMENT}

The author wishes to thank Richard J. Smith for valuable discussions during the development and testing of this TRAC-P update. 


\section{Appendix A}

\section{Listing of LABPRG Input-Data File LABRGH and Update ZERORGH}

\section{File LABRGH}

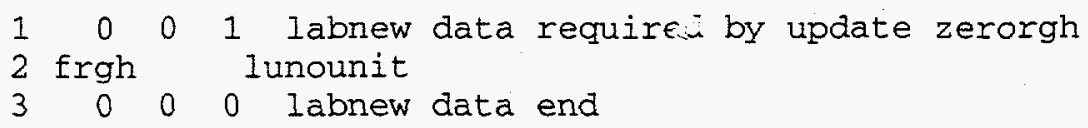

\section{File ZERORGH}

1 *ident zerorgh

$2 * 1$ - 2 02-96--

Code developer: Bob steinke

Date developed: $7 / 28 / 97$

Acknowledgements: KAPL requested a multiplier for the RGH term

Update reviewer: Rich Smith

Date reviewed: $8 / \mathrm{xx} / 97$

Update to be applied to MOD2 _X_ or other :

CFS file path: /073417

Purpose of update: Update zerorgh (ZERO the RGH term) defines a new Namelist variable FRGH that is applied as a multiplier to the gravity-head RGH term in all motion equations evaluated by TRAC-P. The FRGH default value is 1.0 . |FRGH|.LE.1000.0 constrains its userdefined input value.

Basis for update: Allows the TRAC-P user to apply a FRGH multiplier to the gravity-head term in all the motion equations evaluated by TRAC-P. $\mathrm{FRGH}=0.0$ eliminates the gravity-head term from the motions equations.

Dependencies on other updates: none

Justification of non-ANSI coding: N.A.

Is this a NULI update? YES _ $\mathrm{X}_{-}$NO

Does this update generate new unit labels? YES_X_NO If yes, path and /name of LABNEW file:/073417/labrgh

Does the TRAC I/O change because of this update? YES _ X NO TRAC I/O includes input, dump, output, and graphics files. If yes, then submit the necessary manual changes.

New Namelist variable FRGH needs the following format description defined on page $6-8$ in chapter 6 of the TRAC User's Guide:

FRGH : -1000.0 Multiplier applied to the gravity- 1.0 to 1000.0 head term in all motion equations

Does this change require that any of the TRAC support codes, e.g. 
$49 * /$ EXTRACT, GOCNVRT, or EXCON, be changed? YES _ NO _ X_

50 * If yes, then include a description of the necessary changes.

51 */ This description will be passed onto the custodian of the TRAC

$52 * 1$

$53 * 1$

$54 * 1$

$55 * 1$

$56 * 1$

$57 * 1$

$58 * 1$

$59 * 1$

$60 * 1$

$61 * 1$

$62 * 1$

$63 * 1$

$64 * 1$

$65 * 1$

$66 * 1$

$67 * 1$

$68 * 1$

$69 * 1$

$70 * 1$

$71 * 1$

$72 * 1$

$73 * 1$

$74 * 1$

$75 * 1$

$76 * 1$

$77 * 1$

$78 * 1$

$79 * 1$

$80 * 1$

$81 * 1$

$82 * 1$

$83 * 1$

$84 * 1$

$85 * 1$

$86 * 1$

$87 * 1$

$88 * 1$

$89 * 1$

support codes.

Does the TRAC User's Guide, Theory Manual, or Programmer's Guide change because of this update? YES _ $X_{\text {_ }}$ NO

If yes, then submit the necessary manual changes.

See FRGH format description above for the TRAC User's Guide.

NAME OF

SUBROUTINE

OR COMDECK

CHANGED

DESCRIPTION OF CHANGE

CONTRLLR

Stores Namelist variable FRGH in common block CONTRL.

BLKDAT

Initializes Namelist variable $\mathrm{FRGH}=1.0$.

FEMOM

Applies the Namelist variable multiplier FRGH to the gravity-head term in the motion equation of $1 \mathrm{D}$

hydraulic components during the prep stage.

FEMOMX

Applies the Namelist variable multiplier FRGH to the gravity-head term in the xr-direction motion equation of the VESSEL component during the prep stage.

FEMOMY

Applies the Namelist variable multiplier FRGH to the gravity-head term in the yt-direction motion equation of the VESSEL component during the prep stage.

FEMOMZ

Applies the Namelist variable multiplier FRGH to the gravity-head term in the $z$-direction motion equation of the VESSEL component during the prep stage.

INPUT

Defines and echoes to output Namelist variable FRGH.

NAMLST

Tests that Namelist variable FRGH lies in the value range from -1000.0 to 1000.0 .

TF1DS1 Applies the Namelist variable multiplier FRGH to the gravity-head term in the motion equation of $1 D$ hydraulic components during the outer stage.

TF3DS1 Applies the Namelist variable multiplier FRGH to the gravity-head term in all three direction motion equations of the VESSEL component during the

$95 * /$ comdeck contrlir changes

96 *delete upencyl.4 outer stage.

* ercemx,epss , etime,frgh ,htlosi,htloso,

$99 * /$ subroutine blkdat changes

100 *delete fixfr.1

blkdat

101 data alp2, alpcut, alpctr,alp3/1.,0.98,0.995,0.9999/

102

$103 * 1$

104 */ subroutine femom changes

105 *delete fernom.723, femom.724 


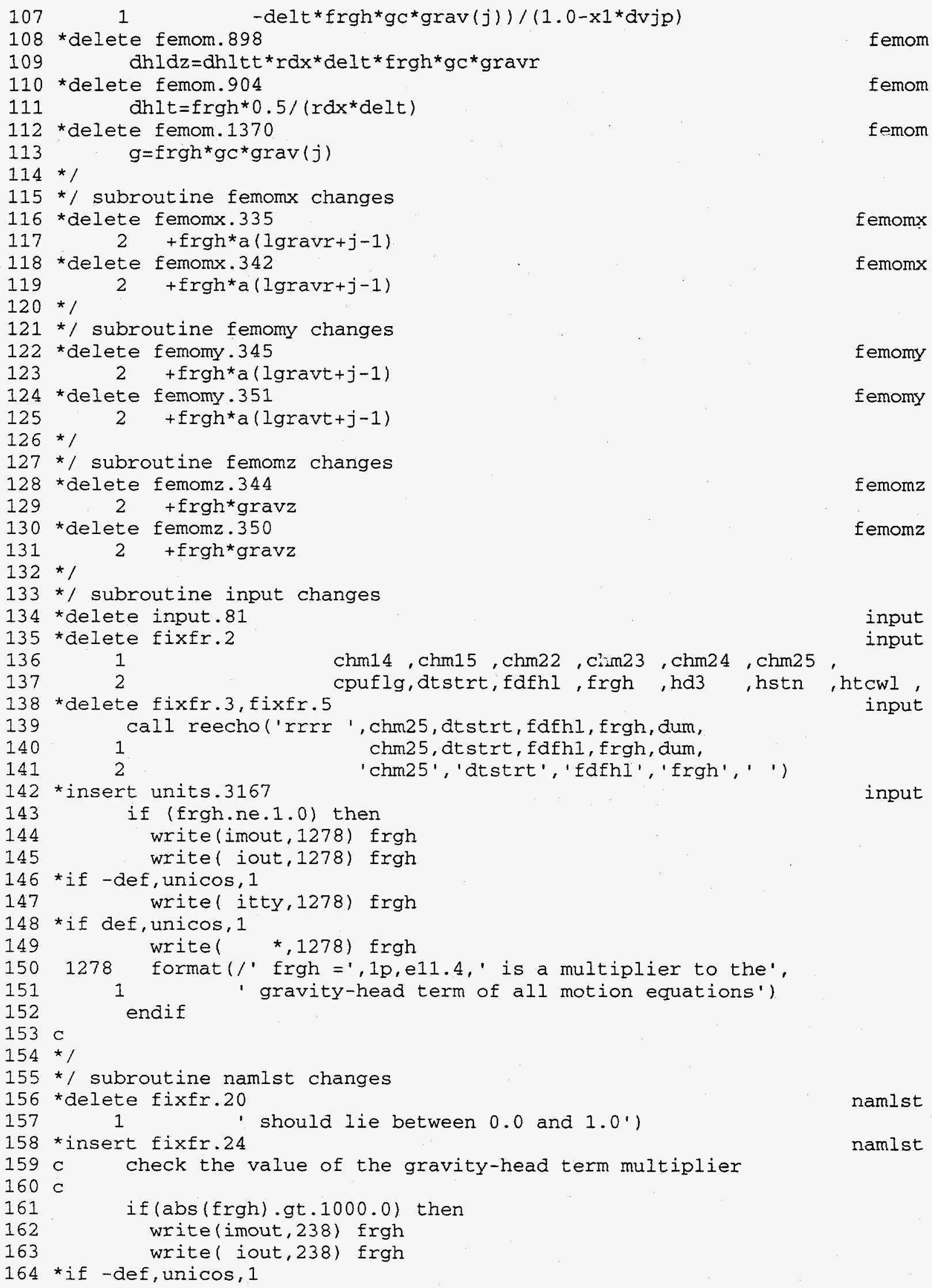




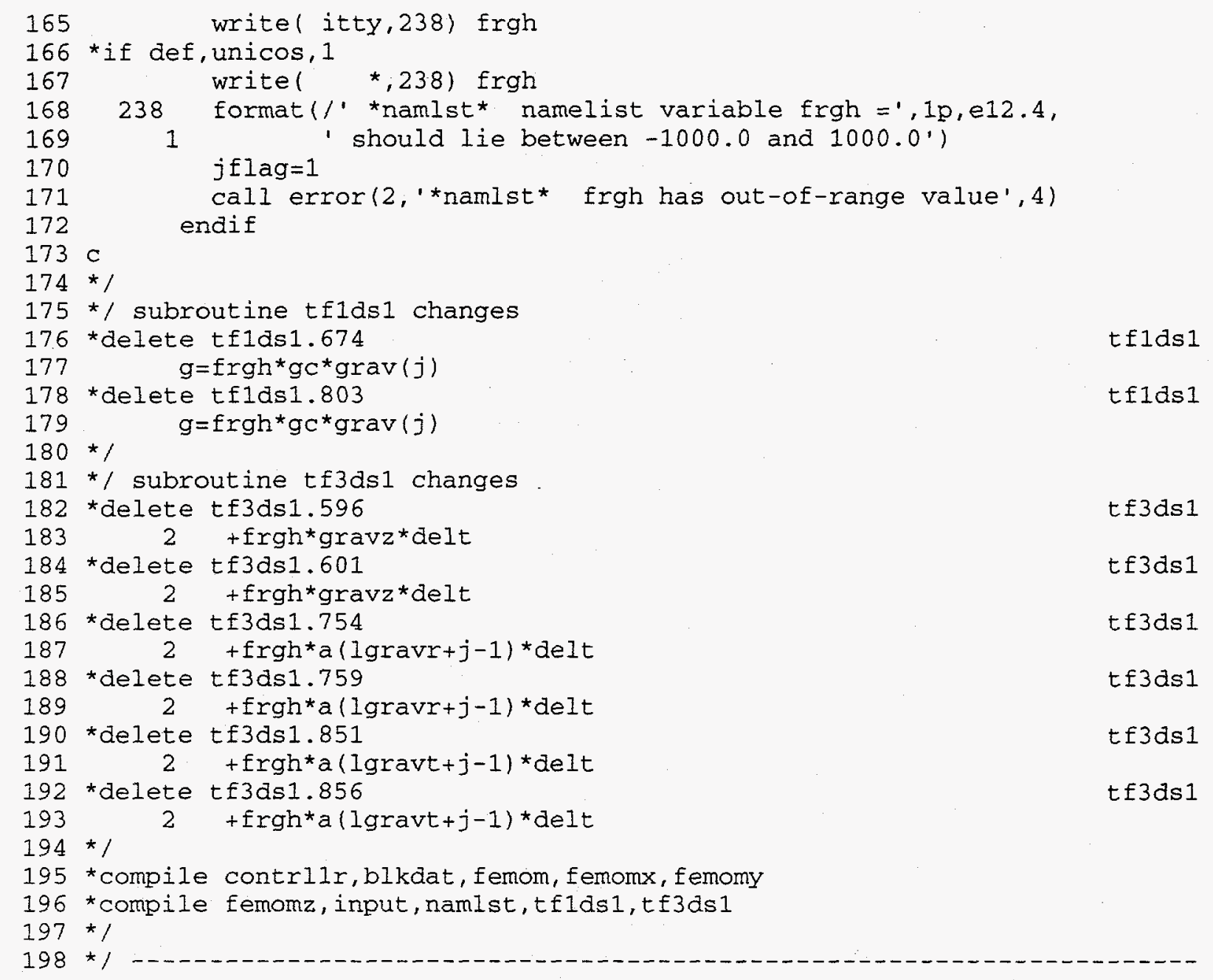




\title{
Appendix B \\ ZIONPWR Test Problem Results
}

\section{ZPWR1IN (ZIONPWR File with Namelist Variable FRGH $=1.0$ Defined by Default)}

\author{
castor:/export/castorl/rgs/trac> cp zionpwr zpwrlin \\ castor:/export/castorl/rgs/trac> cp zpwrlin tracin
}

\section{Terminal Output from Test Problem ZPWR1}

castor:/export/castor1/rgs/trac> trac

input data is being processed

h20 properties are used

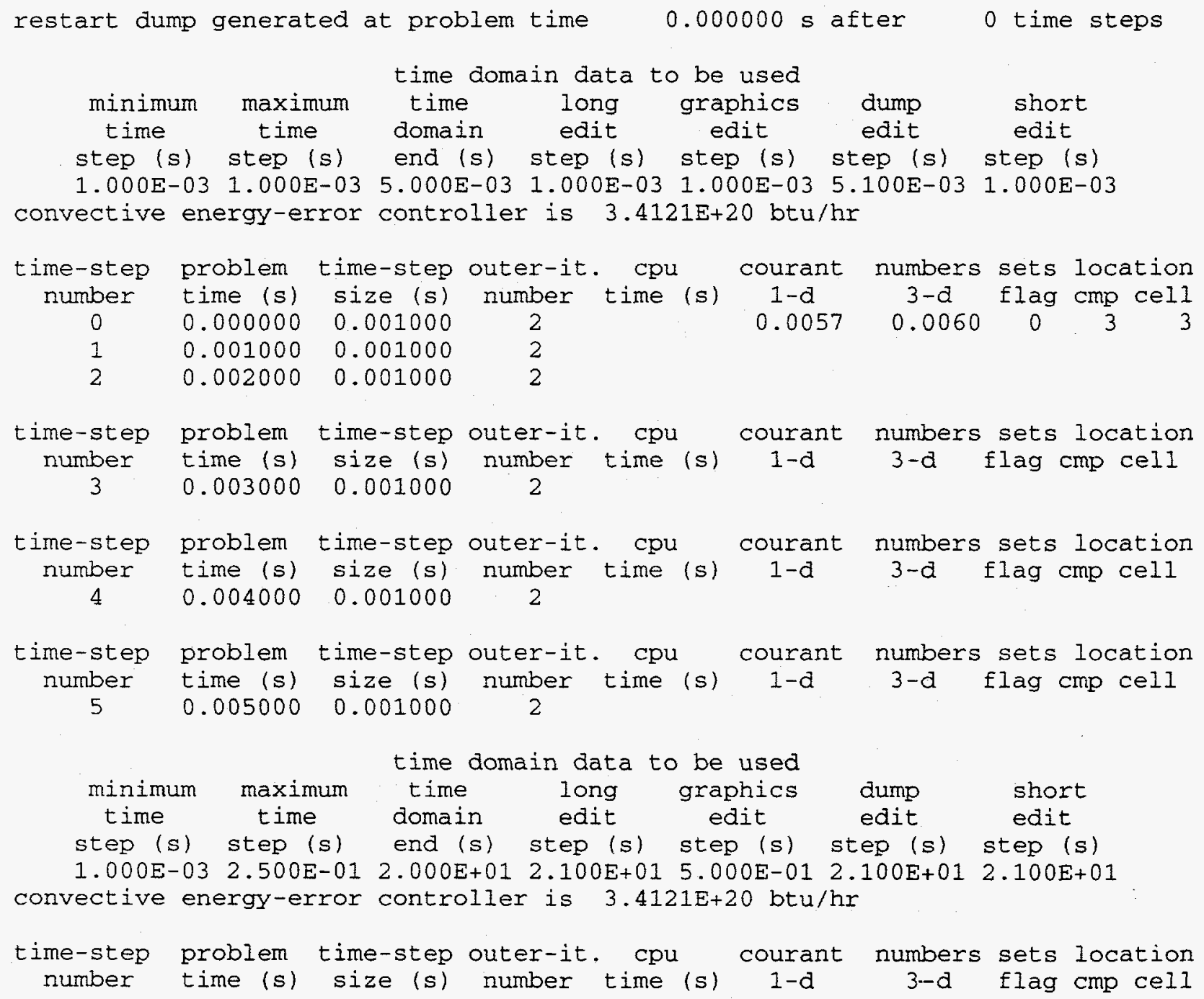




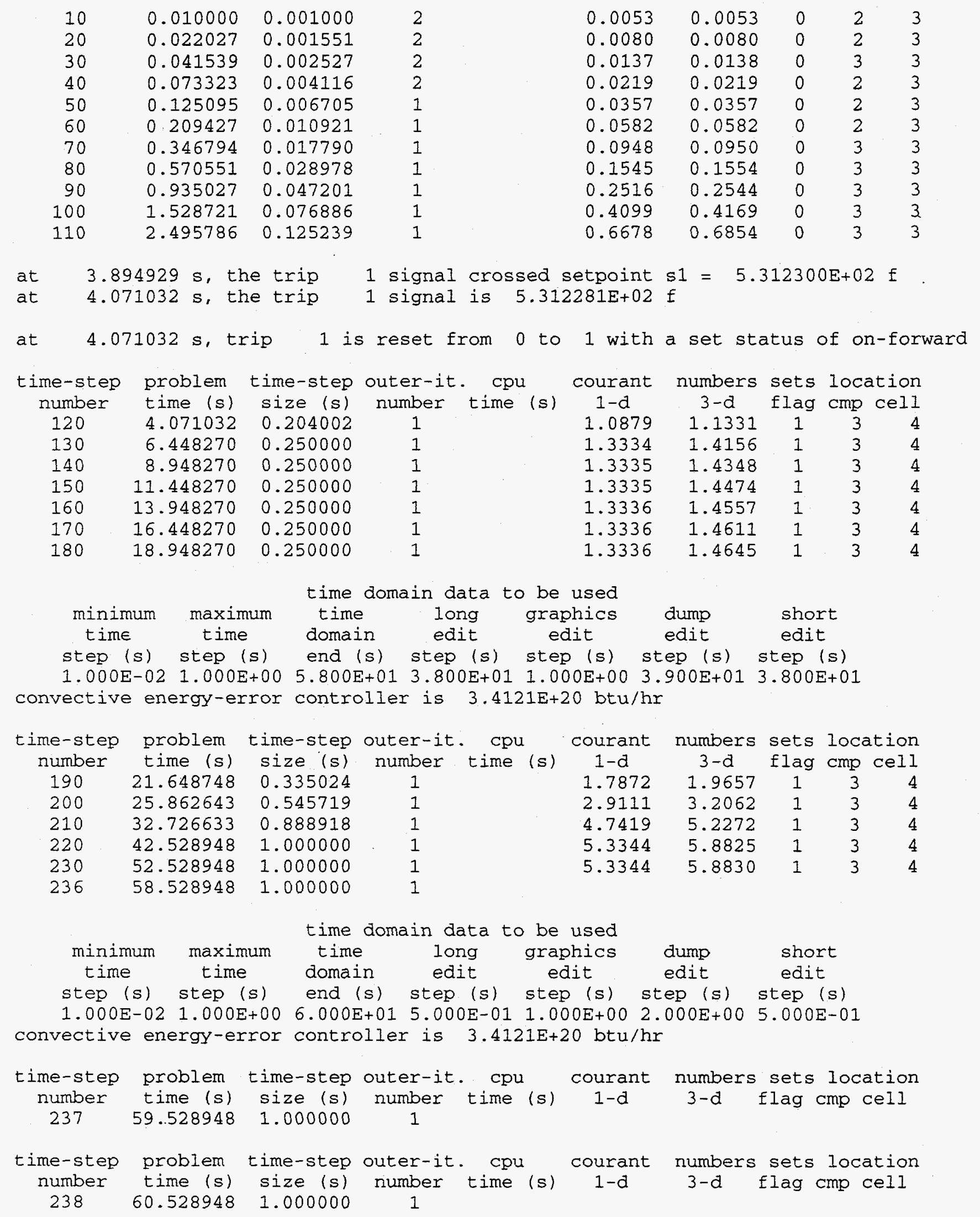




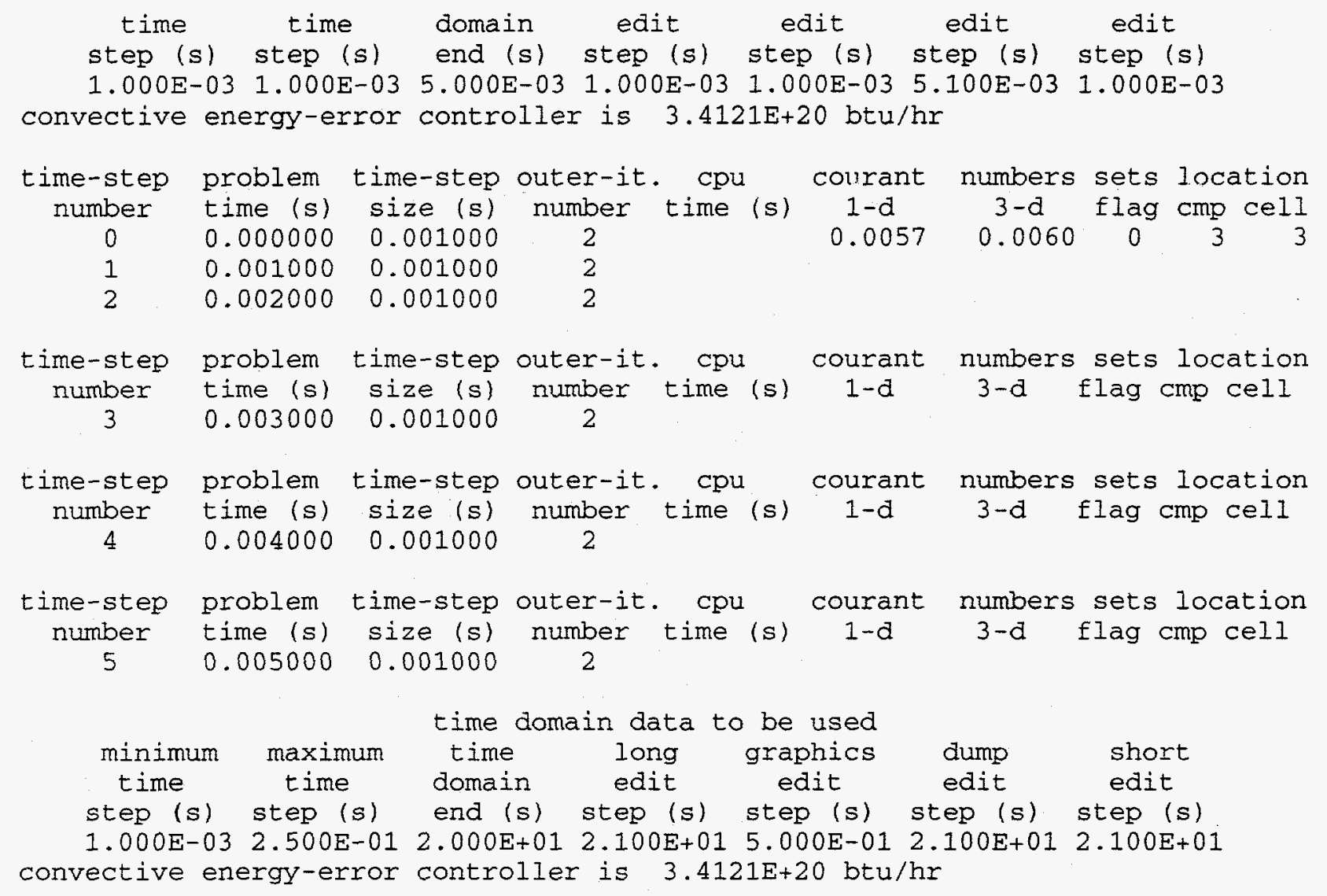

\begin{tabular}{|c|c|c|c|c|c|c|c|c|c|}
\hline $\begin{array}{c}\text { time-step } \\
\text { number }\end{array}$ & $\begin{array}{l}\text { problem } \\
\text { time (s) }\end{array}$ & $\begin{array}{c}\text { time-step } \\
\text { size (s) }\end{array}$ & $\begin{array}{l}\text { outer-it } \\
\text { number }\end{array}$ & $\cdot \begin{array}{c}\text { cpu } \\
\text { time (s) }\end{array}$ & $\begin{array}{c}\text { courant } \\
1-d\end{array}$ & $\begin{array}{c}\text { numbers } \\
3-d\end{array}$ & $\begin{array}{l}\text { sets } \\
\text { flag }\end{array}$ & $\begin{array}{l}\text { loca } \\
\text { cmp }\end{array}$ & $\begin{array}{l}\text { ation } \\
\text { cell }\end{array}$ \\
\hline 10 & 0.010000 & 0.001000 & 2 & & 0.0053 & 0.0053 & 0 & 2 & 3 \\
\hline 20 & 0.022027 & 0.001551 & 2 & & 0.0080 & 0.0080 & 0 & 2 & \\
\hline 30 & 0.041539 & 0.002527 & 2 & & 0.0137 & 0.0138 & 0 & 3 & \\
\hline 40 & 0.073323 & 0.004116 & 2 & & 0.0219 & 0.0219 & 0 & 2 & \\
\hline 50 & 0.125095 & 0.006705 & 1 & & 0.0357 & 0.0357 & 0 & 2 & \\
\hline 60 & 0.209427 & 0.010921 & 1 & & 0.0582 & 0.0582 & 0 & 2 & \\
\hline 70 & 0.346794 & 0.017790 & 1 & & 0.0948 & 0.0950 & 0 & 3 & \\
\hline 80 & 0.570551 & 0.028978 & 1 & & 0.1545 & 0.1554 & 0 & 3 & \\
\hline 90 & 0.935027 & 0.047201 & 1 & & 0.2516 & 0.2544 & 0 & 3 & \\
\hline 100 & 1.528721 & 0.076886 & 1 & & 0.4099 & 0.4169 & 0 & 3 & \\
\hline 110 & 2.495786 & 0.125239 & 1 & & 0.6678 & 0.6854 & 0 & 3 & \\
\hline
\end{tabular}

\begin{abstract}
at $\quad 3.894929 \mathrm{~s}$, the trip 1 signal crossed setpoint sl $=5.312300 \mathrm{E}+02 \mathrm{f}$ at $4.071032 \mathrm{~s}$, the trip 1 signal is $5.312281 \mathrm{E}+02 \mathrm{f}$
\end{abstract}

at $4.071032 \mathrm{~s}$, trip 1 is reset from 0 to 1 with a set status of on-forward

\begin{tabular}{|c|c|c|c|c|c|c|c|c|c|}
\hline $\begin{array}{l}\text { time-step } \\
\text { number }\end{array}$ & $\begin{array}{l}\text { problem } \\
\text { time (s) }\end{array}$ & $\begin{array}{l}\text { time-step } \\
\text { size (s) }\end{array}$ & $\begin{array}{c}\text { outer-it } \\
\text { number }\end{array}$ & $\begin{array}{c}\text { cpu } \\
\text { time (s) }\end{array}$ & $\begin{array}{c}\text { courant } \\
1-d\end{array}$ & $\begin{array}{c}\text { numbers } \\
3-d\end{array}$ & $\begin{array}{l}\text { sets } \\
\text { flag }\end{array}$ & $\begin{array}{l}\text { loca } \\
\text { cmp }\end{array}$ & $\begin{array}{l}\text { tion } \\
\text { cell }\end{array}$ \\
\hline 120 & 4.071032 & 0.204002 & 1 & & 1.0879 & 1.1331 & 1 & 3 & 4 \\
\hline 130 & 6.448270 & 0.250000 & 1 & & 1.3334 & 1.4156 & 1 & 3 & 4 \\
\hline 140 & 8.948270 & 0.250000 & 1 & & 1.3335 & 1.4348 & 1 & 3 & 4 \\
\hline 150 & 11.448270 & 0.250000 & 1 & & 1.3335 & 1.4474 & 1 & 3 & 4 \\
\hline 160 & 13.948270 & 0.250000 & 1 & & 1.3336 & 1.4557 & 1 & 3 & 4 \\
\hline 170 & 16.448270 & 0.250000 & 1 & & 1.3336 & 1.4611 & 1 & 3 & 4 \\
\hline 180 & 18.948270 & 0.250000 & 1 & & 1.3336 & 1.4645 & 1 & 3 & 4 \\
\hline
\end{tabular}




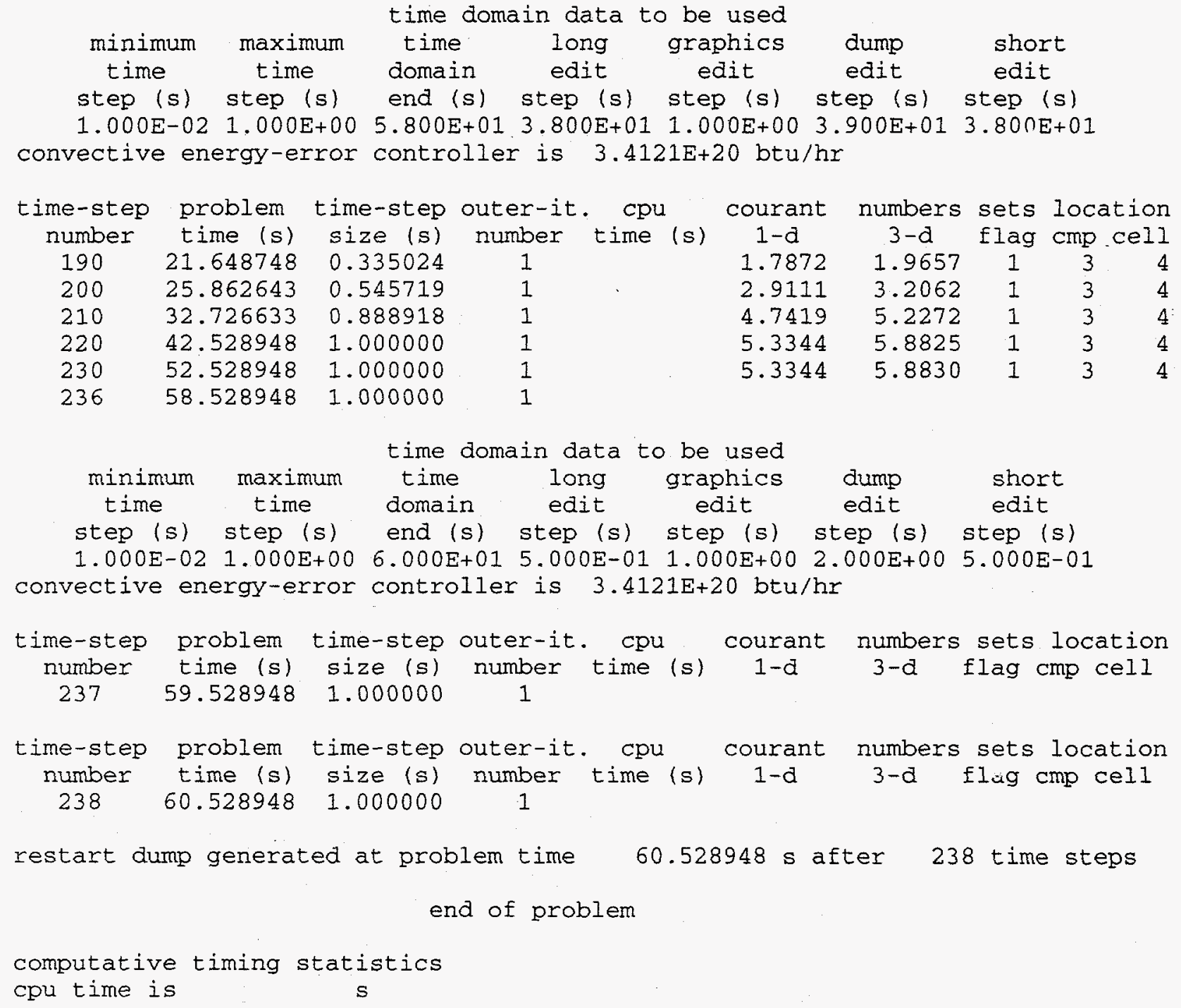




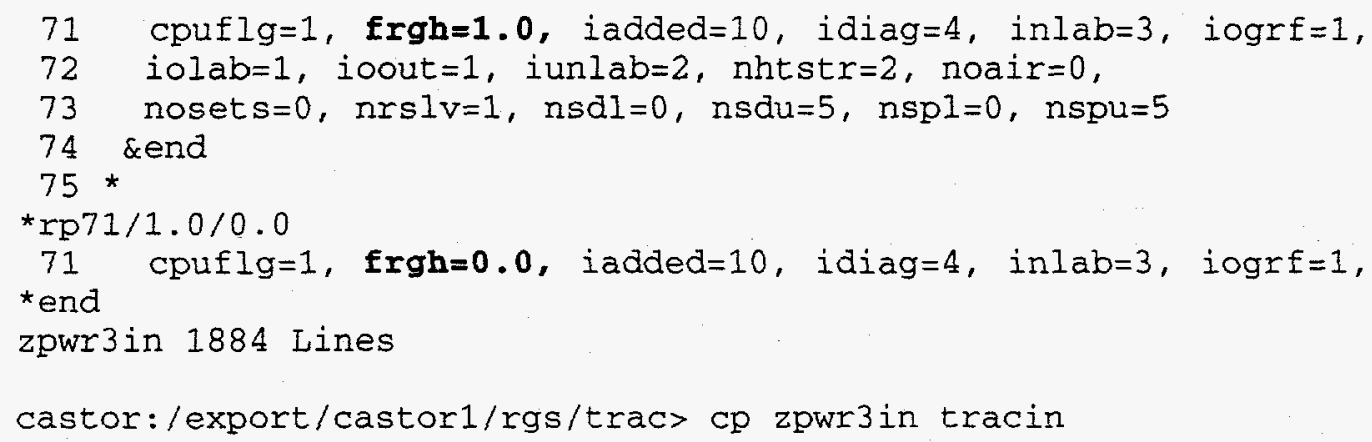

\title{
Terminal Output from Test Problem ZPWR3
}

castor:/export/castor1/rgs/trac> trac

\author{
input data is being processed \\ h2o properties are used
}

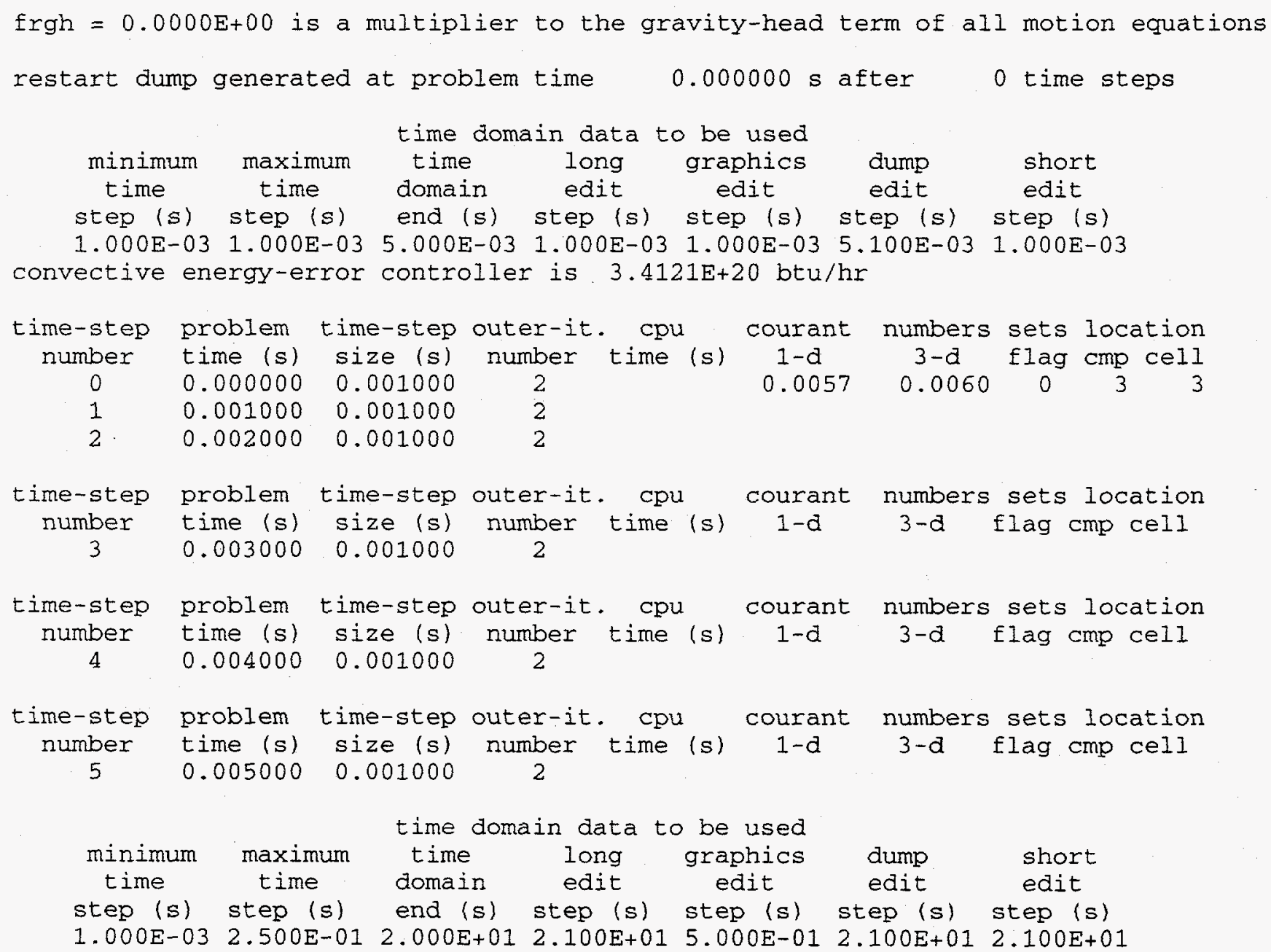




\begin{tabular}{|c|c|c|c|c|c|c|c|c|}
\hline time-step & problem & time-step & outer-it & $\mathrm{cpu}$ & courant & numbers & sets & $10 \mathrm{Ca}$ \\
\hline number & time (s) & size (s) & number & time (s) & $1-d$ & $3-d$ & flag & cmp ce \\
\hline 10 & 0.010000 & 0.001000 & 2 & & 0.0053 & 0.0053 & 0 & 2 \\
\hline 20 & 0.022027 & 0.001551 & 2 & & 0.0078 & 0.0078 & 0 & 2 \\
\hline 30 & 0.041539 & 0.002527 & 2 & & 0.0137 & 0.0138 & 0 & 3 \\
\hline 40 & 0.073323 & 0.004116 & 2 & & 0.0217 & 0.0217 & 0 & 2 \\
\hline 50 & 0.125095 & 0.006705 & 1 & & 0.0355 & 0.0355 & 0 & 2 \\
\hline 60 & 0.209427 & 0.010921 & 1 & & 0.0578 & 0.0578 & 0 & 2 \\
\hline 70 & 0.346794 & 0.017790 & 1 & & 0.0941 & 0.0942 & 0 & 3 \\
\hline 80 & 0.570551 & $0: 028978$ & 1 & & 0.1534 & 0.1541 & 0 & 3 \\
\hline 90 & 0.935027 & 0.047201 & 1 & & 0.2499 & 0.2523 & 0 & 3 \\
\hline 100 & 1.528721 & 0.076886 & 1 & & 0.4070 & 0.4136 & 0 & 3 \\
\hline 110 & 2.495786 & 0.125239 & 1 & & 0.6631 & 0.6798 & 0 & 3 \\
\hline
\end{tabular}

$\begin{array}{ll}\text { at } & 2.881992 \mathrm{~s} \text {, the trip } 1 \text { signal crossed setpoint } \mathrm{s} 1=5.312300 \mathrm{E}+02 \mathrm{f} \\ \text { at } & 2.890603 \mathrm{~s} \text {, the trip } 1 \text { signal is } 5.312289 \mathrm{E}+02 \mathrm{f}\end{array}$

at $\quad 2.890603 \mathrm{~s}$, trip 1 is reset from 0 to 1 with a set status of on-forward

time-step problem time-step outer-it. cpu courant numbers sets location number time (s) size (s) number time (s) $1-d$ 3-d flag cmp cell

$\begin{array}{lllllllll}120 & 4.071032 & 0.204002 & 1 & 1.0802 & 1.1232 & 1 & 3 & 3 .\end{array}$

$\begin{array}{lllllllll}130 & 6.448270 & 0.250000 & 1 & 1.3240 & 1.3989 & 1 & 3 & 3\end{array}$

$\begin{array}{lllllllll}140 & 8.948270 & 0.250000 & 1 & 1.3240 & 1.4176 & 1 & 3 & 4\end{array}$

$\begin{array}{lllllllll}150 & 11.448270 & 0.250000 & 1 & 1.3241 & 1.4299 & 1 & 3 & 4\end{array}$

$\begin{array}{lllllllll}160 & 13.948270 & 0.250000 & 1 & 1.3241 & 1.4380 & 1 & 3 & 4\end{array}$

$\begin{array}{lllllllll}170 & 16.448270 & 0.250000 & 1 & 1.3241 & 1.4433 & 1 & 3 & 4\end{array}$

$\begin{array}{lllllllll}180 & 18.948270 & 0.250000 & 1 & 1.3241 & 1.4467 & 1 & 3 & 4\end{array}$

\begin{tabular}{ccccc} 
& \multicolumn{2}{c}{ time domain data to be used } \\
minimum maximum time long graphics dump & short \\
time & time domain edit & edit & edit & edit
\end{tabular}

step (s) step (s) end (s) step (s) step (s) step (s) step (s)

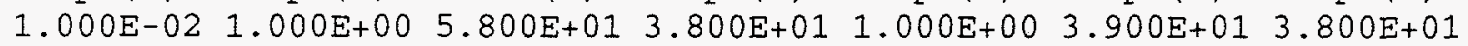

convective energy-error controller is $3.4121 \mathrm{E}+20 \mathrm{btu} / \mathrm{hr}$

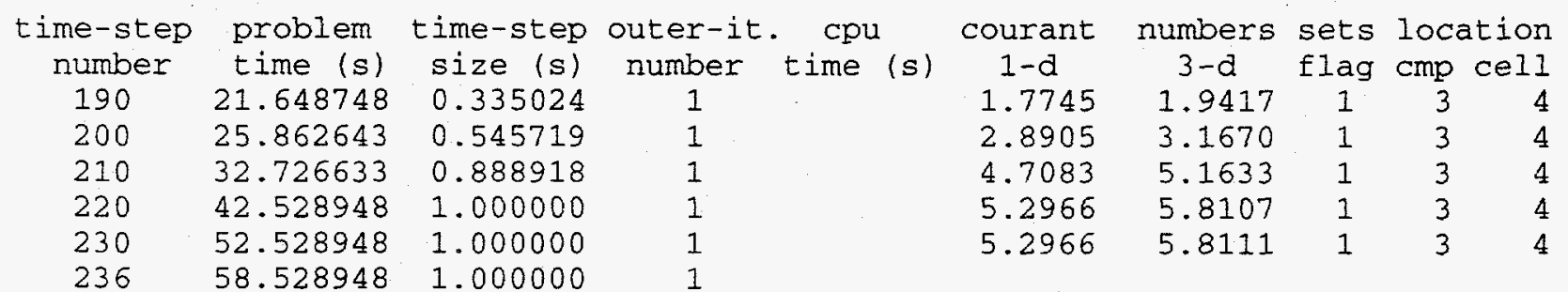

\begin{tabular}{|c|c|c|c|c|c|}
\hline & & cime & dató & to be used & \\
\hline $\begin{array}{l}\text { minimum } \\
\text { time }\end{array}$ & $\begin{array}{l}\text { maximum } \\
\text { time }\end{array}$ & $\begin{array}{l}\text { time } \\
\text { domain }\end{array}$ & $\begin{array}{l}\text { long } \\
\text { edit }\end{array}$ & $\begin{array}{l}\text { graphics } \\
\text { edit }\end{array}$ & $\begin{array}{l}\text { dump } \\
\text { edit }\end{array}$ \\
\hline
\end{tabular}

step (s) step (s) end (s) step (s) step (s) step (s) step (s)

$\begin{array}{lllllll}1.000 \mathrm{E}-02 & 1.000 \mathrm{E}+00 & 6.000 \mathrm{E}+01 & 5.000 \mathrm{E}-01 & 1.000 \mathrm{E}+00 & 2.000 \mathrm{E}+00 & 5.000 \mathrm{E}-01\end{array}$

convective energy-error controller is $3.4121 \mathrm{E}+20 \mathrm{btu} / \mathrm{hr}$

time-step problem time-step outer-it. cpu courant numbers sets location number time (s) size (s) number time (s) 1-d $3-d$ flag cmp cell $\begin{array}{llll}237 & 59.528948 & 1.000000 & 1\end{array}$ 


$$
\begin{aligned}
& \begin{array}{ccccc}
\text { time-step problem time-step outer-it.l cpu } & \text { courant numbers sets location } \\
\text { number } & \text { time (s) } & \text { size (s) number time (s) } & 1-d & 3-d \text { flag cmp cell } \\
238 & 60.528948 & 1.000000 & 1
\end{array} \\
& \text { restart dump generated at problem time } 60.528948 \mathrm{~s} \text { after } 238 \text { time steps } \\
& \text { end of problem }
\end{aligned}
$$

computative timing statistics

cpu time is

$\mathrm{s}$

\section{ZPWR3OUT (TRCOUT File Results from Test Problem 3 for Later Comparison with ZPWR4OUT)}

castor:/export/castor1/rgs/trac> mv trcout zpwr3out

ZPWR4IN (ZIONPWR with Namelist Variable FRGH $=1.0$ input and 1D-component GRAVs and 3D-component GZC Reset from 1.0 to 0.0 )

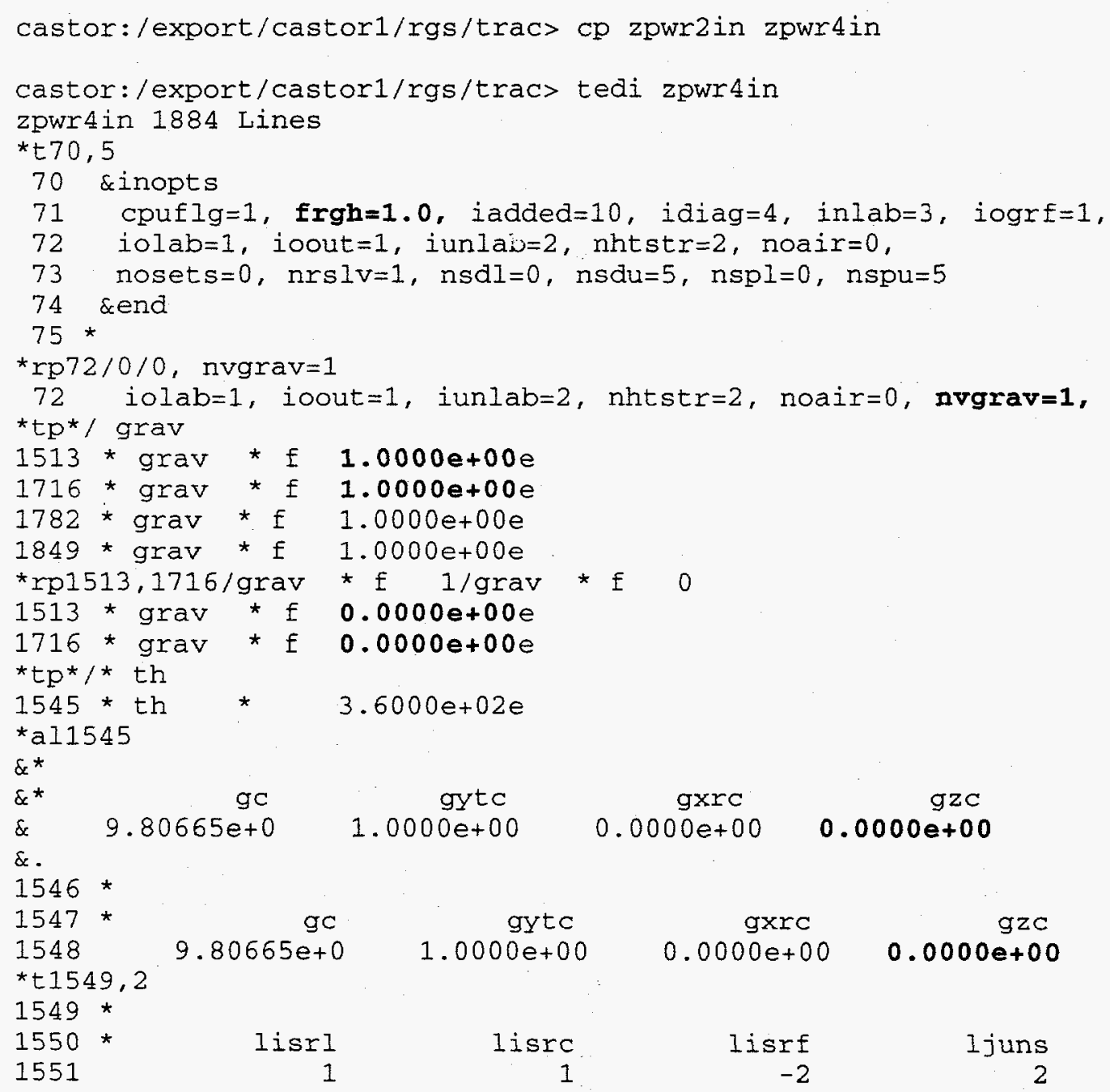


zpwr4in 1887 Lines

Note: GRAVs on IInes 1782 and 1849 are for HTSTRs ROD 6 and SLAB 7

castor:/export/castor1/rgs/trac> cp zpwr4in tracin

\section{Terminal Output from Test Problem ZPWR4}

castor: /export/castor1/rgs/trac> trac

input data is being processed

h2o properties are used

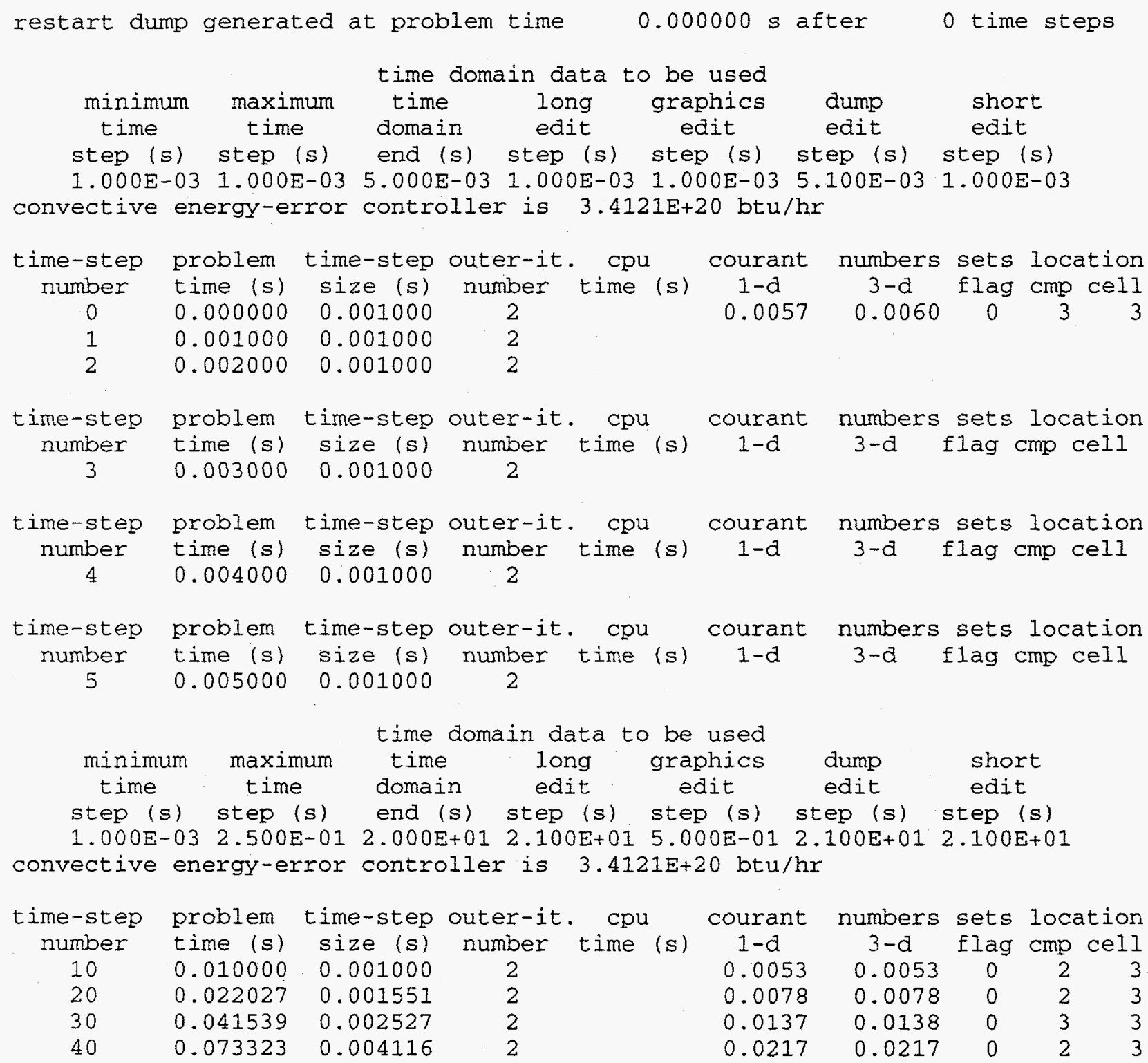




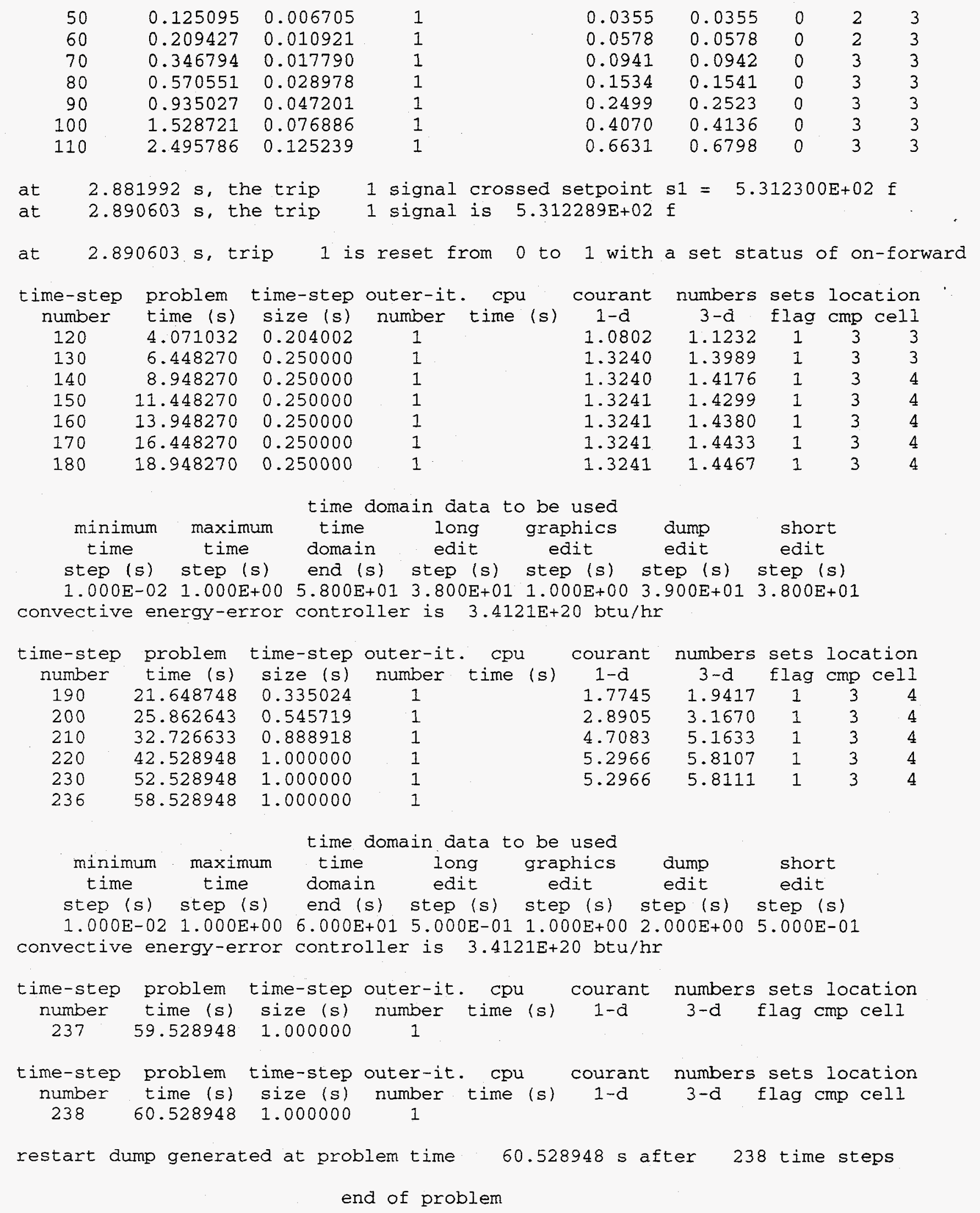


computative timing statistics

cpu time is

$s$

\section{ZPWR4OUT (TRCOUT File Results from Test Problem 4)}

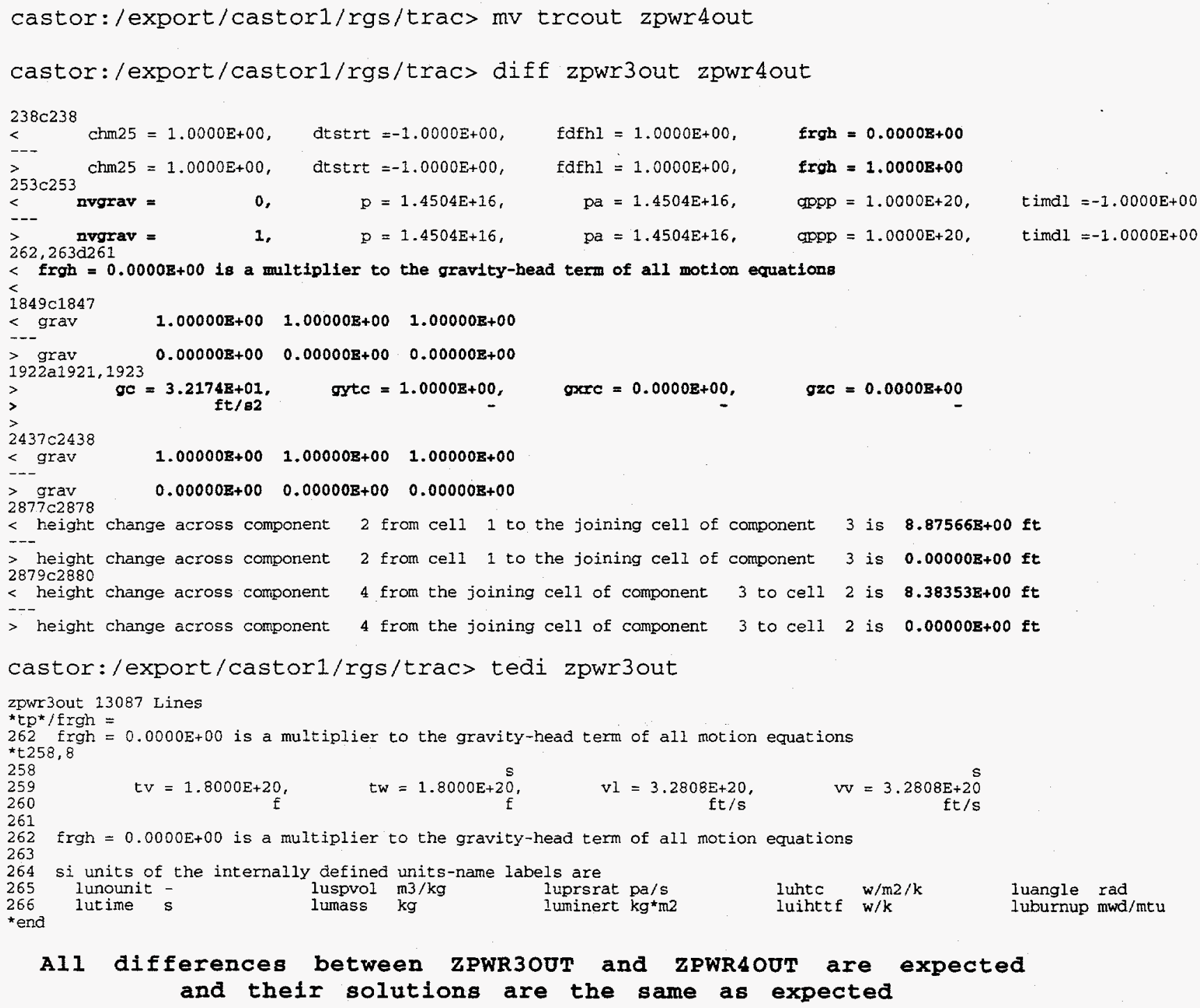

\section{ZPWR5IN (ZIONPWR File with Namelist Variable FRGH = 0.6 Input)}

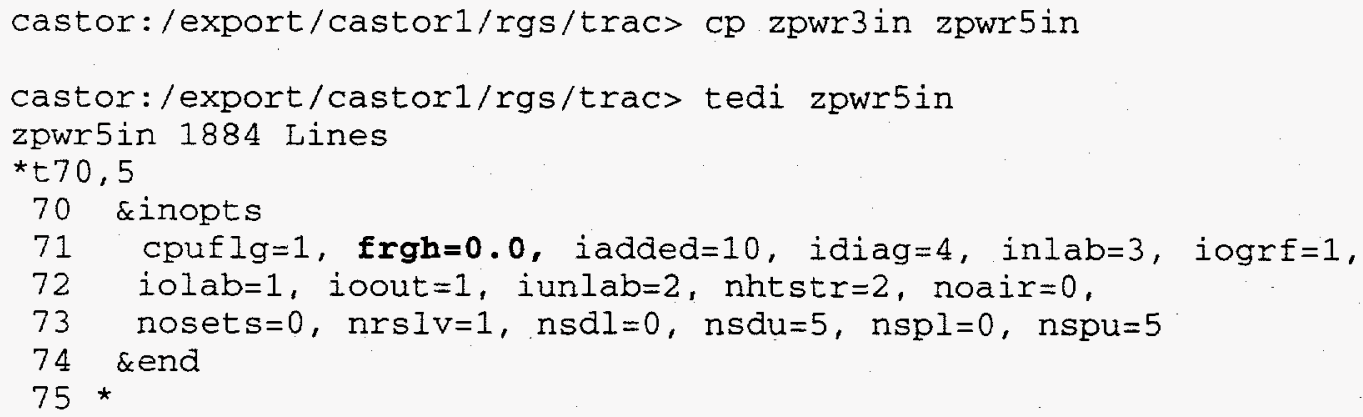




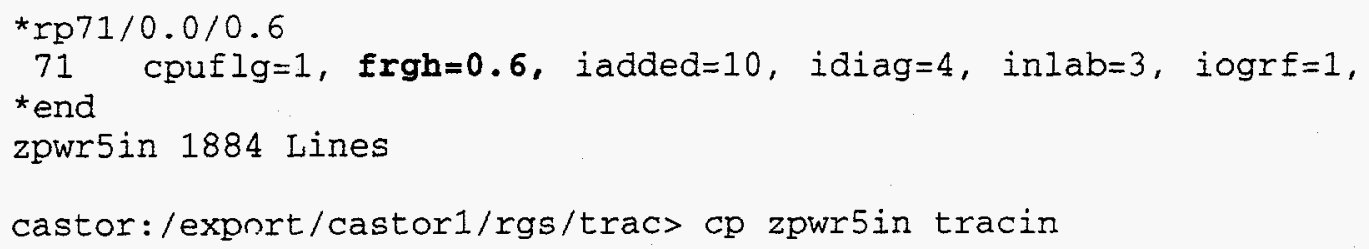

\title{
Terminal Output from Test Problem ZPWR5
}

castor:/export/castor1/rgs/trac> trac

\author{
input data is being processed \\ h2o properties are used
}

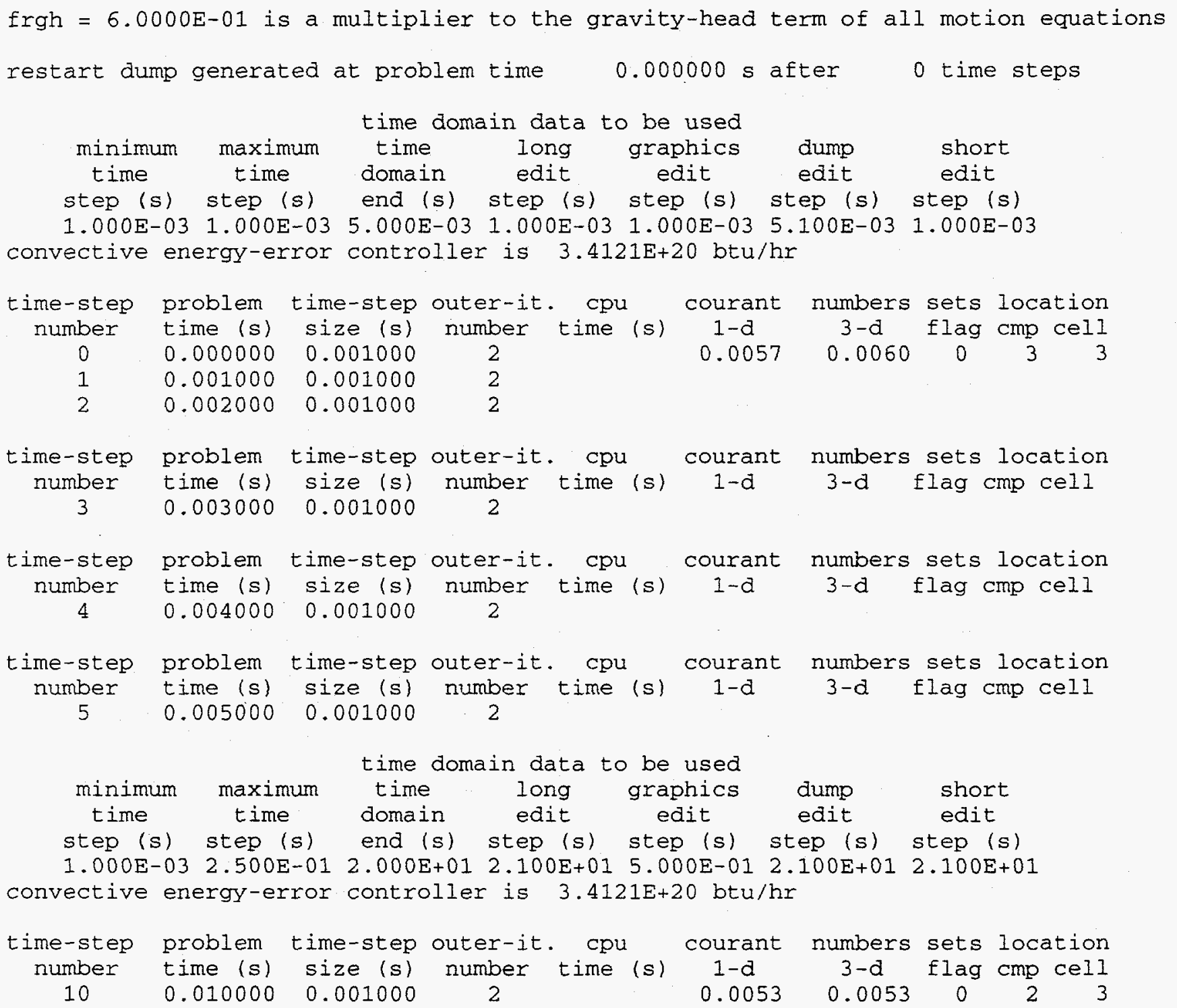




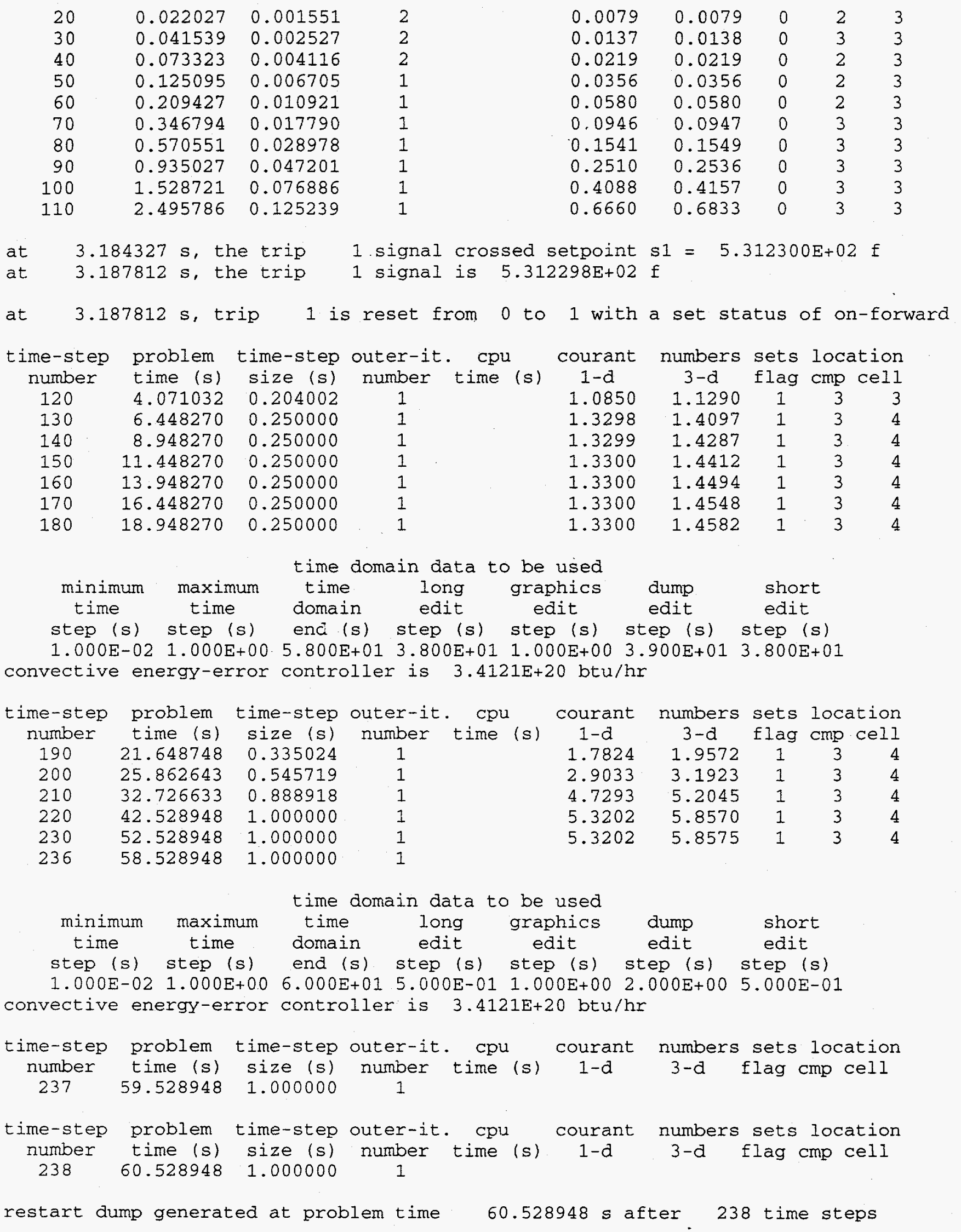


end of problem

computative timing statistics

cpu time is

$s$

ZPWR5OUT (TRCOUT File Results from Test Problem 5 for Later Comparison with ZPWR6OUT)

castor:/export/castor1/rgs/trac> mv trcout zpwr5out

ZPWR6IN (ZIONPWR with Namelist Variable FRGH $=1.0$ input and 1D-component

GRAVs and 3D-component GZC Reset from 0.0 to 0.6 and -0.6 )

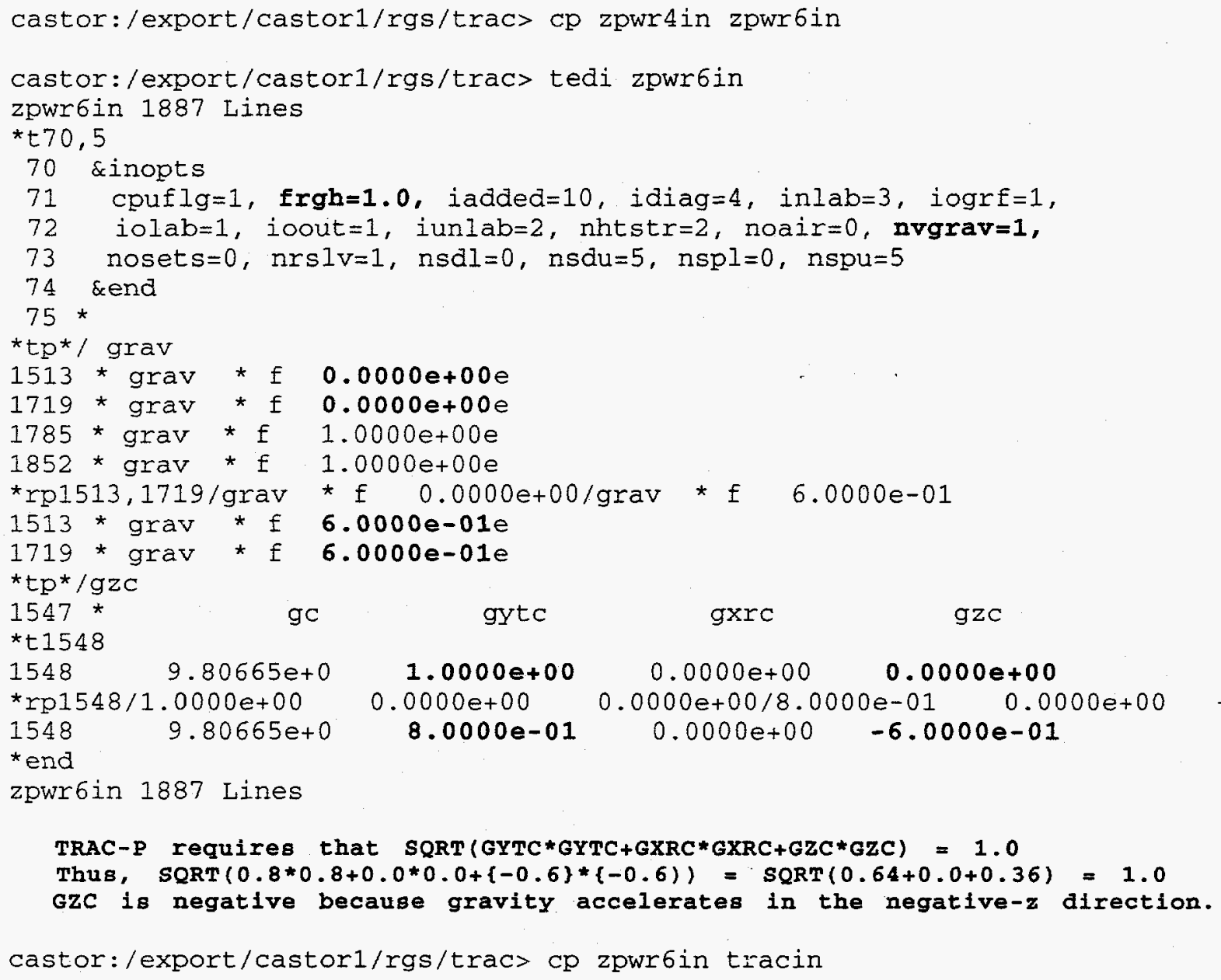

Terminal Output from Test Problem ZPWR6

castor:/export/castor1/rgs/trac> trac 
input data is being processed

h20 properties are used

restart dump generated at problem time $0.000000 \mathrm{~s}$ after 0 tine steps

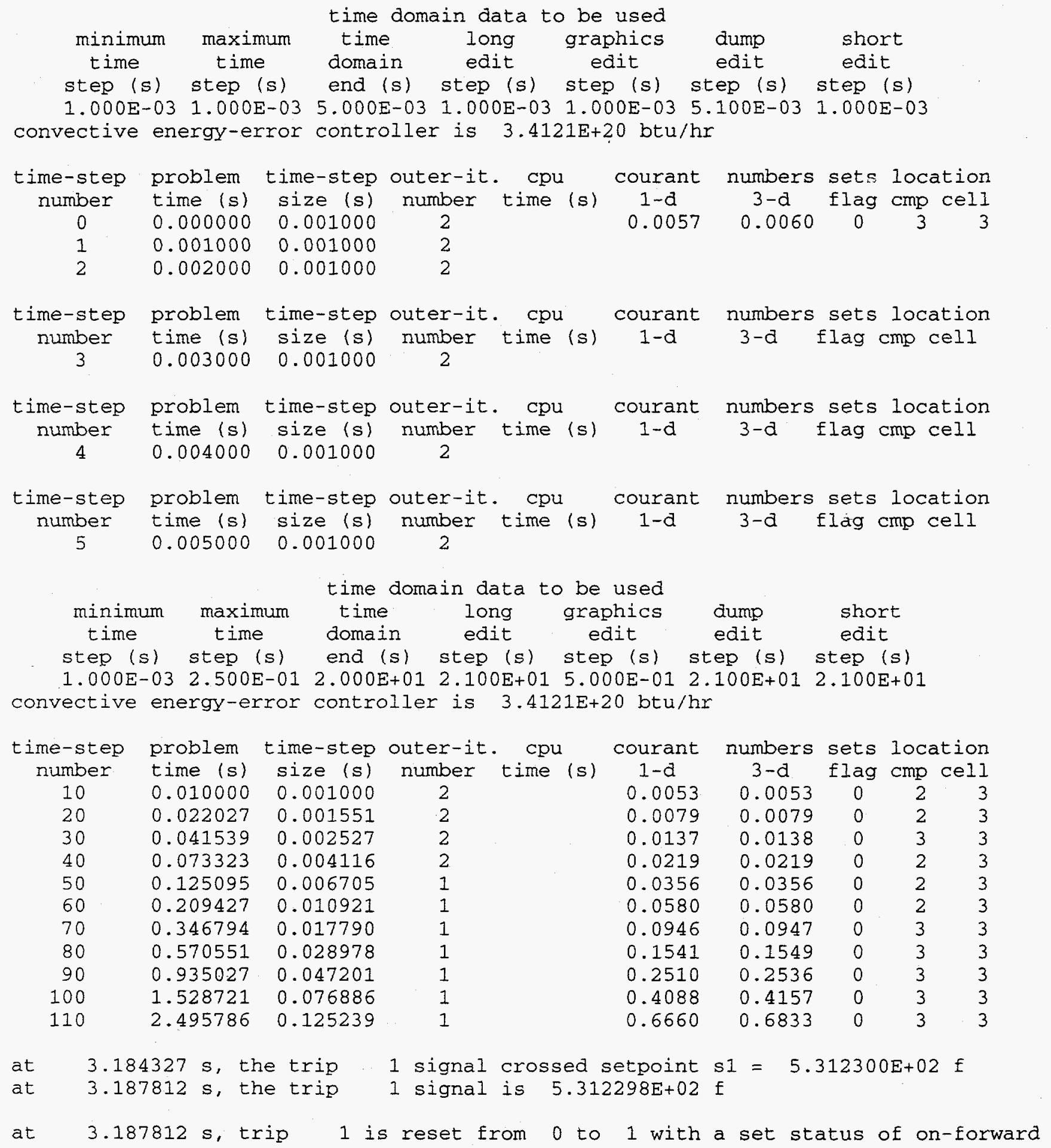




\begin{tabular}{|c|c|c|c|c|c|c|c|c|}
\hline $\begin{array}{c}\text { time-step } \\
\text { number }\end{array}$ & $\begin{array}{l}\text { problem } \\
\text { time (s) }\end{array}$ & $\begin{array}{c}\text { time-step } \\
\text { size (s) }\end{array}$ & $\begin{array}{c}\text { outer-it } \\
\text { number }\end{array}$ & $\begin{array}{l}\text { cpu } \\
\text { time (s) }\end{array}$ & $\begin{array}{c}\text { courant } \\
1-d\end{array}$ & $\begin{array}{c}\text { numbers } \\
3-d\end{array}$ & $\begin{array}{l}\text { sets } \\
\text { flag }\end{array}$ & $\begin{array}{l}\text { loca } \\
\text { cmp }\end{array}$ \\
\hline 120 & 4.071032 & 0.204002 & 1 & & 1.0850 & 1.1290 & 1 & 3 \\
\hline 130 & 6.448270 & 0.250000 & 1 & & 1.3298 & 1.4097 & 1 & 3 \\
\hline 140 & 8.948270 & 0.250000 & 1 & & 1.3299 & 1.4287 & 1 & 3 \\
\hline 150 & 11448270 & 0.250000 & 1 & & 1.3300 & 1.4412 & 1 & 3 \\
\hline 160 & 13.948270 & 0.250000 & 1 & & 1.3300 & 1.4494 & 1 & 3 \\
\hline 170 & 16.448270 & 0.250000 & 1 & & 1.3300 & 1.4548 & 1 & 3 \\
\hline 180 & 18.948270 & 0.250000 & 1 & & 1.3300 & 1.4582 & 1 & 3 \\
\hline
\end{tabular}

minimum maximum time domain data to be used
time time long graphics dump short
step (s) step (s) end (s) step (s) step (s) step (s) step (s)

$1.000 \mathrm{E}-02 \quad 1.000 \mathrm{E}+00 \quad 5.800 \mathrm{E}+01 \quad 3.800 \mathrm{E}+01 \quad 1.000 \mathrm{E}+00 \quad 3.900 \mathrm{E}+01 \quad 3.800 \mathrm{E}+01$ convective energy-error controller is $3.4121 \mathrm{E}+20 \mathrm{btu} / \mathrm{hr}$

\begin{tabular}{|c|c|c|c|c|c|c|c|c|c|}
\hline time-step & problem & time-step & outer-it & cpu & courant & numbers & sets & 100 & \\
\hline number & time (s) & size (s) & number & time (s) & $1-d$ & $3-a$ & flag & $\mathrm{cmp}$ & \\
\hline 190 & 21.648748 & 0.335024 & 1 & & 1.7824 & 1.9572 & 1 & 3 & \\
\hline 200 & 25.862643 & 0.545719 & 1 & & 2.9033 & 3.1 & 1 & 3 & \\
\hline 210 & 32.726633 & 0.888918 & 1 & & 4.7293 & 5.2045 & 1 & 3 & \\
\hline 220 & 42.5 & 1.000000 & 1 & & 5.3202 & 5.8570 & 1 & 3 & \\
\hline 230 & 52.5 & 1.000000 & 1 & & 5.3202 & 5.8575 & 1 & 3 & \\
\hline 236 & 58.528948 & 1.000000 & 1 & & & & & & \\
\hline
\end{tabular}

\footnotetext{
time domain data to be used

$\begin{array}{ccccc}\text { minimum maximum time long graphics dump } & \text { short } \\ \text { time } & \text { time domain edit } & \text { edit } & \text { edit } & \text { edit }\end{array}$

step (s) step (s) end (s) step (s) step (s) step (s) step (s)

$\begin{array}{lllllll}1.000 E-02 & 1.000 E+00 & 6.000 E+01 & 5.000 E-01 & 1.000 E+00 & 2.000 E+00 & 5.000 E-01\end{array}$

convective energy-error controller is $3.4121 \mathrm{E}+20 \mathrm{btu} / \mathrm{hr}$

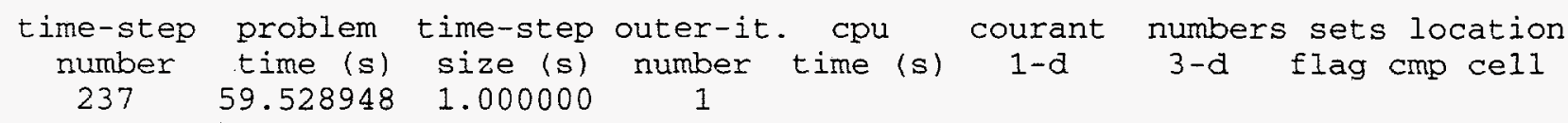

time-step problem time-step outer-it. cpu courant numbers sets location number time (s) size (s) number time (s) $1-d$ d-d flag cmp cell $\begin{array}{llll}238 & 60.528948 & 1.000000 & 1\end{array}$

restart dump generated at problem time $60.528948 \mathrm{~s}$ after 238 time steps

end of problem

computative timing statistics

cpu time is $s$
}

\section{ZPWR6OUT (TRCOUT File Results from Test Problem 6)}

castor:/export/castorl/rgs/trac> mv trcout zpwr6out

castor:/export/castor1/rgs/trac> diff zpwr5out zpwr6out

$\begin{array}{lllll}238 \mathrm{c} 238 & \operatorname{chm} 25=1.0000 \mathrm{E}+00, & \text { dtstrt }=-1.0000 \mathrm{E}+00, & \text { fdfhl }=1.0000 \mathrm{E}+00, & \text { frgh }=6.0000 \mathrm{E}-01 \\ -- & \operatorname{chm} 25=1.0000 \mathrm{E}+00, & \text { destrt }=-1.0000 \mathrm{E}+00, & \text { fdfhl }=1.0000 \mathrm{E}+00, & \text { frgh }=1.0000 \mathrm{E}+00\end{array}$ 


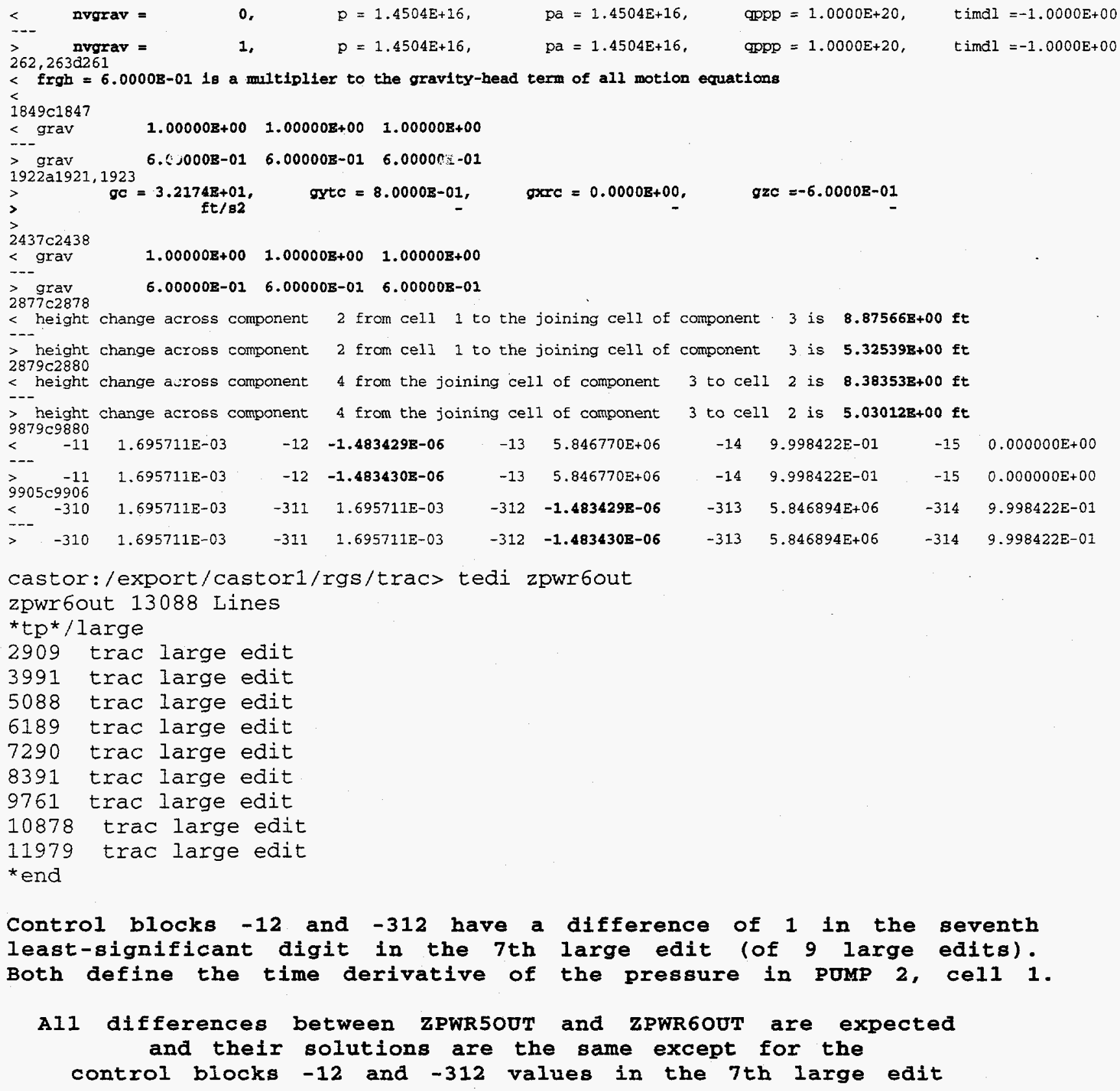

